\title{
FONTES DE CÉLULAS-TRONCO HEMATOPOÉTICAS PARA TRANSPLANTES
}

\author{
SOURCES OF HEMATOPOIETIC STEM CELLS FOR TRANSPLANTATION
}

Wellington Azevedo \& Maria Cecília C Ribeiro²

\footnotetext{
${ }^{1}$ Diretor do Serviço de Transplante de Medula Óssea do Hospital das Clínicas da Universidade Federal de Minas Gerais. ${ }^{2}$ Médica Hematologista.

CorResPondênCIA: Av.Brasil, 691/901 - Bairro São Lucas. CEP: 30.140-000 - Belo Horizonte - MG. e-mail: azevedow@medicina.ufmg.br
}

AZEVEDO W \& RIBEIRO MCC. Fontes de células-tronco hematopoéticas para transplantes. Medicina, Ribeirão Preto, 33: 381-389, out./dez. 2000.

RESUMO: Atualmente, vários tipos de células-tronco hematopoéticas (CTH), provenientes de diferentes tecidos e doadores, são disponíveis para transplante em seres humanos. Nesta revisão, discute-se a utilização de CTH alogênicas, obtidas diretamente da medula óssea ou mobilizadas para o sangue periférico com fatores de crescimento hematopoéticos. Comparam-se os dois tipos de transplante em relação a enxertia, recaída da doença básica, ocorrência de doença do enxerto-contra-hospedeiro (GVHD) aguda e crônica e sobrevida. Embora haja nítida vantagem do transplante de CTH periféricas, a curto prazo, em relação à medula óssea, a maior incidência de GVHD crônico e o seguimento ainda curto dos pacientes não recomenda, por ora, a adoção generalizada do TCTH de sangue periférico para transplantes alogênicos.

UNITERMOS: Transplante de Medula Óssea. Fatores de Crescimento de Células Hematopoiéticas. Células Hematopoiéticas Primitivas.

\section{INTRODUÇÃO}

O transplante de células-tronco hematopoéticas (TCTH) é, hoje, reconhecidamente, um tratamento efetivo para doenças neoplásicas, hematológicas ou não, doenças metabólicas e deficiências imunológicas.

O TCTH pode ser dividido em três tipos, dependendo do doador das células progenitoras:

1. Alogênico: quando as células progenitoras são provenientes de um doador geneticamente distinto, seja HLA compatível ou não, relacionado ou não. Em algumas situações, é preciso a substituição completa da hematopoese do paciente pela de um doador saudável, habitualmente um familiar com compatibilidade no sistema HLA. Este tipo de transplante apresenta complicações relacionadas a rea- ções imunológicas entre receptor e doador, desde a rejeição até a doença do enxerto-contra-hospedeiro (DECH ou GVHD). As reações imunológicas atuam, também, sobre as células neoplásicas residuais - efeito antitumor ( graft-versus leukemia ou GVL) - que contribui para a menor tendência de recaída neste tipo de transplante.

2. Autólogo: quando as células progenitoras utilizadas são do próprio paciente. Este procedimento se realiza, fundamentalmente, em doenças neoplásicas, tanto hematológicas, quanto em alguns tipos de tumores sólidos (vide Capítulo IX deste Simpósio). Em determinadas fases destas doenças, podem estar indicadas altas doses de quimioterapia com o objetivo de erradicar completamente a doença de base. Esta quimioterapia tão intensa pro- 
duz, normalmente, aplasia medular irreversível ou muito prolongada. A reinfusão de células progenitoras, coletadas previamente, reduz o tempo de aplasia medular e reestabelece a hematopoese, minimizando as complicações do tratamento. As células progenitoras são obtidas de forma programa$\mathrm{da}$, quando o paciente se encontra em remissão completa da doença, exceto no caso do Mieloma Múltiplo em que, apesar de a medula óssea estar infiltrada de plasmócitos, eles não são oncogênicos, e haverá redução da massa tumoral e aumento da sobrevida. Este tipo de tansplante tem menos risco que o alogênico por não existir nenhum tipo de reação imunológica entre receptor e doador; por isso, pode ser usado em pacientes mais idosos. Entretanto, como o efeito antitumoral é menor, o índice de recidivas é maior.

3. Singênico: quando realizado entre gêmeos idênticos. A identidade antigênica entre doador e receptor é absoluta e não se produz, portanto, nenhuma complicação imunológica. Mas, não existirá o efeito antitumoral, podendo haver recidivas.

Já em relação aos tipos de células progenitoras, contamos, atualmente, com as seguintes opções:

1- Células da medula óssea

2- Células-tronco do sangue periférico (CTP)

3- Células de sangue do cordão umbilical (veja capítulo III deste Simpósio)

4- Células de fígado fetal

5- Células cultivadas

6- Células geneticamente modificadas

A medula óssea e as células-tronco periféricas podem ser usadas tanto em transplantes autólogos quanto em alogênicos, assim como as células cultivadas e geneticamente modificadas, e podem ser usadas a fresco ou congeladas. Já as células de sangue de cordão umbilical e de fígado fetal são destinadas exclusivamente aos transplantes alogênicos.

O transplante de células-tronco hematopoéticas periféricas é bem aceito como forma de tratamento de doenças malignas ou não. Desde o início dos anos 90, as CTP, colhidas por aférese após a mobilização com fatores de crescimento de colônias de granulócitos ou de granulócitos-macrófagos (G-CSF ou GMCSF), têm sido largamente usadas como fonte de células progenitoras hematopoéticas para o transplante autólogo $\mathrm{o}^{(1)}$ e levam à recuperação medular (número de células polimorfonucleadas maior que $500 / \mathrm{mm}^{3}$ e número de plaquetas maior que $20.000 /$ $\mathrm{mm}^{3}$ ) mais rápida, quando comparadas com a $\mathrm{MO}^{(2)}$. As CTP também têm sido coletadas de doadores saudáveis, após a mobilização com fatores de crescimento, e usadas como alternativa para substituir a $\mathrm{MO}$ nos transplantes alogênicos ${ }^{(3)}$ e também levam à recuperação medular mais rápida, quando comparadas com a $\mathrm{MO}^{(3 / 6)}$. Entretanto, ainda há dúvidas sobre qual a melhor fonte de células progenitoras a ser usada neste tipo de transplante, já que outros fatores, além da recuperação medular, estão envolvidos e devem ser analisados para se obter a resposta.

Este capítulo dedica-se à discussão das duas fontes mais utilizadas nos transplantes alogênicos, a medula óssea (MO) e as células-tronco periféricas. Serão analisados, criticamente, os trabalhos da literatura, incluindo os do autor, comparando as modalidades de transplante em relação à enxertia e rejeição, à ocorrência de doença do enxerto-contra-hospedeiro, à recaída da doença neoplásica e à sobrevida dos pacientes.

\section{RECUPERAÇÃO HEMATOPOÉTICA}

Champlin et al. ${ }^{(3)}$ estudaram 288 pacientes adultos que receberam CTP não manipuladas e HLA idênticas para tratamento de LMA ou LLA em $1^{\mathrm{a}}$ ou $2^{\mathrm{a}}$ remissão ou LMC em fase crônica ou acelerada, entre 1995 e 1996. Usaram, como grupo-controle, 536 pacientes com as mesmas características, só que recebendo MO. Todos os dados dos pacientes provinham do registro internacional de TMO (IBMTR). Os pacientes que receberam CTP recuperaram granulócitos e plaquetas mais rapidamente do que os que recberam MO, com diferença estatisticamente significante.

Do mesmo modo, Vigorito et al. ${ }^{(4)}$, em estudo realizado na UNICAMP com 40 pacientes randomizados para CTP ou MO, todos com doador HLA compatível, com idade entre 10 e 60 anos e doença oncoematológica, observaram, no grupo de CTP, recuperação mais rápida de granulócitos, menor necessidade de transfusões de hemácias e plaquetas, menor consumo de antimicrobianos, menor tempo de internação e de nutrição parenteral. Entretanto, provavelmente devido ao número reduzido de pacientes, essas diferenças não alcançaram significância estatística, o que só ocorreu com a recuperação da contagem de plaquetas (Tabela I). 


\begin{tabular}{|c|c|c|c|}
\hline & TMO & TCTHP & $\mathrm{P}$ \\
\hline Dia NAN $>0,5 \times 10^{9} / \mathrm{L}$ & $+18(13-30)$ & $+16(11-25)$ & $0,15^{\star}$ \\
\hline Dia plaquetas $>20 \times 10^{9} / \mathrm{L}$ & $+17(10-40)$ & $+12(9-36)$ & $0,01^{*}$ \\
\hline № unidades plaquetas transfundidas & $18(1-341)$ & $13(4-44)$ & 0,32 \\
\hline № unidades de $\mathrm{CH}$ transfundidas & $5(1-75)$ & $4(2-12)$ & 0,20 \\
\hline Dia de internação & $+26(18-48)$ & $+21(18-42)$ & 0,08 \\
\hline Antibióticos (dias) & $19(8-41)$ & $16,5(9-32)$ & 0,21 \\
\hline Anfotericina B (dose total mg) & $568,75(187-750)$ & $362,50(105-825)$ & 0,41 \\
\hline NPP (dias) & $11(0-33)$ & $9(0-36)$ & 0,16 \\
\hline \multicolumn{4}{|c|}{$\begin{array}{l}\text { NAN = Número Absoluto de Neutrófilos; } \mathrm{CH}=\text { Concentrado de Hemácias } \\
\text { NPP = Nutrição Parenteral Prolongada. } \\
\text { `Breslow's test } \\
\text { Adaptada da ref.(4). }\end{array}$} \\
\hline
\end{tabular}

Heldal et al. ${ }^{(5)}$ realizaram estudo randomizado com 61 pacientes com LMA e LLA, LMC, mielofibrose primária e mielodisplasia com idade entre $15 \mathrm{e}$ 60 anos e com doador HLA idêntico, ou com até um antígeno incompatível, e observaram recuperação mais rápida, tanto nos granulócitos, como nas plaquetas, com diferença estatisticamente significante em favor do grupo que recebeu CTP.

Russel et al. ${ }^{(7)}$ compararam 87 pacientes com LMA e mielodisplasia, com doadores HLA compatíveis, que receberam, entre 1987 e 1998, transplantes com CTP ou MO. A recuperação de granulócitos e de plaquetas foi mais rápida nos pacientes que receberam CTP, ambas com diferença estatisticamente significante, tanto no grupo de melhor prognóstico (LMA $1^{\mathrm{a}}$ remissão - $\mathrm{p}<0,0001$ para as duas linhagens) como no de pior prognóstico (LMA $2^{\mathrm{a}}$ remissão e mielodisplasia - $\mathrm{p}<0,0001$ para granulócitos e $\mathrm{p}<0,04$ para plaquetas).

Levine et al. ${ }^{(8)}$, em trabalho envolvendo 24 crianças com idade entre nove meses e 17 anos, que receberam CTP HLA idênticas para tratamento de doenças malignas (LMA, LLA, LMC e Mielodisplasia) e não malignas (Talassemia e Síndromes de Kostmann e de Wiskott-Aldrich), também observaram recuperação medular mais rápida quando comparada com MO.
A recuperação medular mais rápida é creditada ao maior número de células-tronco (CD34+) existentes na CTP.

\section{DECH AGUDA E CRÔNICA}

Nos mesmos trabalhos relatados acima, foi observado, em relação à ocorrência de doença do enxerto-contra-hospedeiro:

1. risco de DECH aguda, graus II a IV, similar nos grupos de CTP e MO, mesmo tendo sido observado que o número de células T é maior em 1 log na CTP;

2. risco de DECH crônica maior no grupo de CTP.

Há algumas particularidades nos trabalhos:

1. Vigorito et al. ${ }^{(4)}$ registraram casos mais graves de DECH crônica e uma maior incidência de acometimento de mucosa oral, no grupo de CTP (Tabelas II e III);

2. Russel et al. observaram maior incidência de DECH crônica, nos pacientes de melhor prognóstico que receberam CTP, com diferença estatisticamente significante, quando comparados com os de melhor prognóstico que receberam $\mathrm{MO}$, mas não observaram maior índice de DECH crônica na CTP, quando se compararam os grupos de pior prognóstico ${ }^{(7)}$; 


\begin{tabular}{|c|c|c|c|}
\hline & TMO & TCTHP & $\mathrm{P}$ \\
\hline $\mathrm{DECH}-\mathrm{a}$ avaliáveis & 16 & 15 & \\
\hline Grau $2-4$ & $3 / 16(18,7 \%)$ & $4 / 15(26,6 \%)$ & $0,40^{*}$ \\
\hline Grau 3 - 4 & $2 / 3$ & $2 / 4$ & \\
\hline DECH-c avaliáveis & 15 & 14 & \\
\hline Total afetados & $8 / 15(53,3 \%)$ & $10 / 14(71,4 \%)$ & $0,53^{* *}$ \\
\hline Extenso & $4 / 8(50 \%)$ & $10 / 10(100 \%)$ & $0,02^{*}$ \\
\hline Limitado & $4 / 8(50 \%)$ & 0 & $0,02^{*}$ \\
\hline $\begin{array}{l}{ }^{*} \text { Fisher; }{ }^{* *} x^{2} \\
\text { Adaptada da ref.(4). }\end{array}$ & & & \\
\hline
\end{tabular}

Tabela III - Órgãos / ou tecidos envolvidos com clínica de DECH-c entre os pacientes submetidos a TMO ou a TCTH periféricas.

\begin{tabular}{|lccc|}
\hline & TMO & TCTHP & P \\
\hline Pele & $1(12,5 \%)$ & $2(20 \%)$ & 0,50 \\
Fígado & $8(100 \%)$ & $10(100 \%)$ & - \\
Olhos & $1(12,5 \%)$ & $5(50 \%)$ & 0,10 \\
Mucosa oral & $3(37,5 \%)$ & $9(90 \%)$ & 0,03 \\
Pulmão & $1(12,5 \%)$ & 0 & 0,40 \\
\hline Adaptada da ref.(4). & & & \\
\hline
\end{tabular}

3. Levine et al. constaram que os pacientes infantis, que receberam metotrexato como parte do esquema de profilaxia de DECH tiveram menos DECH aguda $^{(8)}$, efeito também observado em crianças transplantadas com $\mathrm{MO}^{(9)}$.

Era de se esperar que os pacientes recebendo 1 log a mais de células T (CD3+) na CTP desenvolvessem mais DECH aguda do que os transplantados com medula óssea. A não ocorrência deste fato, confirmado em vários trabalhos, pode ser explicado por alguns fatores ${ }^{(10,11,12)}$.
1. o G-CSF induz a migração de células T da medula óssea para o sangue periférico do doador e estas são infundidas no paciente; as células T CD4+ ou CD8+, estimuladas pelo G-CSF, produzem, predominantemente, citocinas do tipo 2 (Th 2), secretantes de interleucinas 4 e 10 (IL4 e IL10) que inibem a produção de citocinas do tipo 1 (Th 1), secretantes de interferon-gama e de interleucina 2; as duas últimas estimulam os macrófagos, as células NK e as células T citotóxicas, responsáveis pelas reações imunológicas que levam à DECH, mas, como estão ini- 
bidas pelas Th 2, a incidência de DECH aguda não está aumentada;

2. G-CSF induz uma maior produção de células mononucleares, que também produzem IL10 e que têm efeito de controle inibitório na resposta imune;

3. G-CSF parece, também, atuar numa subpopulação de células $\mathrm{T}$ supressoras naturais (CD4-/CD8-), que inibem a resposta citotóxica durante $\mathrm{DECH}$ aguda e parece também inibir a alorreatividade das células T;

4. G-CSF também aumenta o número de células dendríticas que contribuem para maior produção de IL4 e IL10, e estas são inibidoras de citocinas do tipo 1 que levam à $\mathrm{DECH}$ aguda;

5. é possível que a recuperação medular mais rápida, observada na CTP, diminua a incidência e a gravidade de infecções e que este fato possa funcionar como proteção ao aparecimento de $\mathrm{DECH}$ aguda.

Entretanto, em recente trabalho, apresentado por Przepiorka et al. ${ }^{(13)}$, sobre fatores de risco para DECH aguda, após TCTP, foram evidenciados, pelos autores, como estatisticamente significativos, um maior número de células CD34+ e esquemas menos intensos para profilaxia de $\mathrm{DECH}$. Nesta análise, a inclusão de metotrexato no esquema profilático, junto com ciclosporina, mostrou ser melhor para reduzir a incidência de DECH aguda. Outros fatores, como a doença de base, histocompatibilidade entre doador e re ceptor, compatibilidade entre sexo do doador e do receptor, sorologia positiva do doador para CMV, isoimunização prévia do doador e altas doses de radiação corporal total (TBI), como parte de esquema de condicionamento, não foram estatisticamente significativos para aumentar a incidência de DECH aguda. Foi observada uma tendência maior de DECH aguda em pacientes mais velhos (50 anos), mas sem significância estatística.

Em relação à maior incidência de $\mathrm{DECH}$ crônica, talvez o ponto central para a discussão da melhor fonte de células progenitoras esteja nos processos de alorreatividade e autoreatividade. A alorreatividade, no contexto do GVHDc, é decorrente do aumento da resposta Th 2 (IL4 e IL10), provocada pelo grande número de células $\mathrm{T}$, que continua a existir e que causará danos a órgãos alvos (pele, mucosas, fígado, intestino e pulmão). A autoreatividade produz auto-anticorpos, tais como fatores antinucleares, an- ticorpo anti-musculatura lisa e anti-DNA, presentes em 11 a $62 \%$ dos pacientes com DECH crônica, que, também, poderão causar dano tissular. Como a incidência de DECH aguda é pequena, a maioria dos casos de DECH crônica se comporta como episódio de novo e tem boa resposta ao tratamento imunossupressor ${ }^{(10)}$.

\section{RECAÍDA}

Em relação à recaída, a maioria dos trabalhos não encontrou diferença entre as duas fontes de células progenitoras, mesmo quando se analisa a recaída entre os que tiveram ou não DECH crônica, apesar de todos ressaltarem que, com o seguimento curto, tal afirmação não possa ser feita de forma tão categórica. Entretanto, Korbling et al. ${ }^{(14)}$ mostraram que, na experiência em 100 pacientes recebendo transplantes alogênicos HLA idênticos, no MD Anderson Cancer Center, a recaída foi menor no grupo que recebeu CTP. Elmaagacli et al. ${ }^{(15)}$, analisando 91 pacientes com LMC em fase crônica, que receberam transplantes por CTP e MO, observaram que os pacientes que receberam CTP tiveram menor risco de apresentar doença residual mínima (bcr-abl PCR+). Creditam esse achado ao fato de a CTP possuir mais células progenitoras, promovendo, assim, pega medular mais rápida, o que poderia inibir clones leucêmicos residuais, e ao efeito antitumoral, provocado pelas células T e pelas células NK também contidas em grande número na CTP.

\section{REJEIÇÃO, MORTALIDADE E SOBREVI- DA LIVRE DE DOENÇA}

Nos trabalhos discutidos anteriormente, não foram observadas diferenças estatísticas entre uma fonte e outra de células progenitoras em relação à rejeição, à mortalidade relacionada ao tratamento (MRT) e à sobrevida livre de doença (SLD), a não ser nos trabalhos de Champlin et al. ${ }^{(3)}$ e de Russel et al. ${ }^{(7)}$. Eles mostraram que, em pacientes com doença avançada (LMA $2^{\mathrm{a}}$ remissão e LMC fase acelerada), a MRT foi menor e a SLD foi maior nos pacientes que receberam $\mathrm{CTP}^{(3)}$ e que a SLD, em quatro anos, foi maior para os pacientes de bom prognóstico (LMA $1^{\text {a }}$ remissão) do grupo de $\mathrm{CTP}^{(7)}$, apesar de apresentarem qualidade de vida pior (Karnofsky $<80 \%$ ), provavelmente devido à ocorrência de GVHD crônico. 


\section{ATUALIZAÇÃO DOS DADOS DA LITE- RATURA}

Em recente simpósio - $3^{\text {rd }}$ International Symposium on Allogeneic Peripheral Blood Progenitor Cell Transplantation- Suíça/2000 - vários trabalhos foram apresentados, mostrando os efeitos positivos do transplante, utilizando a CTP:

1. Simpson et al. avaliaram, de janeiro de 1996 a fevereiro de 2000, 228 pacientes portadores de LMC, LMA ou mielodisplasia, submetidos a transplante (119 MO e 109 CTP), e observaram ${ }^{(16)}$ :

- recuperação do número de leucócitos e de plaquetas mais rápida no grupo de CTP;

- em relação à GVHD aguda grau II-IV e GVHD crônica, não houve diferença estatística;

- a taxa de mortalidade no D+100 foi menor no grupo de CTP $(6,7 \%)$ contra $15,5 \%$ no grupo de BM;

- a sobrevida avaliada após 19,5 meses foi maior no grupo de CTP, com diferença estatística.

2. Bensinger et al. observaram, em 172 pacientes submetidos a transplante, no período de março de 1996 a julho de $1999^{(17)}$ :

- pega medular mais rápida, com diferença estatística no grupo que recebeu CTP;

- sem diferença na incidência de GVHD aguda e GVHD crônica;

- sobrevida global e livre de eventos maior no grupo de CTP, com diferença estatística;

- em doença avançada, a sobrevida global e livre de eventos também foi maior no grupo de CTP.

3. Heldal et al., avaliando 61 pacientes portadores de leucemia, mielofibrose ou mielodisplasia, submetidos a transplante, observaram ${ }^{(18)}$ :

- recuperação do número de leucócitos e de plaquetas mais rapidamente no grupo de CTP, com diferença estatística;

- incidência de mortalidade associada ao transplante e sobrevida livre de eventos, semelhante nos grupos de CTP e MO;

- GVHD aguda em seis pacientes que receberam CTP e em três pacientes que receberam MO;

- GVHD crônica em 15 pacientes do grupo de CTP e em oito no grupo de MO.

4. Vigorito et al., já com 60 pacientes no estudo randomizado, iniciado em $1997^{(4)}$, reiteram as observações de que ${ }^{(19)}$ :
- há diferença estatística na recuperação do número de leucócitos e do número de plaquetas a favor do grupo que recebeu CTP;

- não há diferença na incidência de GVHD aguda entre os grupos de CTP e MO;

- em relação à GVHD crônica, a incidência foi maior no grupo de CTP;

- não há diferença na sobrevida global e livre de eventos entre os dois grupos.

5. Guardiola et al. em estudo multicêntrico, envolvendo 229 pacientes portadores de mielodisplasia, que receberam transplante por CTP (95 pacientes) ou por MO (134 pacientes), constataram ${ }^{(20)}$ :

- pega medular mais rápida e incidência de GVHD crônica maior no grupo de CTP;

- sem diferença na mortalidade nos dois grupos;

- recaída menor nos pacientes que receberam CTP, assim como a sobrevida livre de eventos;

- aumento na sobrevida após seis meses do transplante no grupo de CTP.

6. Ringden et al., em estudo envolvendo 107 pacientes recebendo CTP compatível de doador não relacionado, e 107 pacientes recebendo MO também compatível de doador não relacionado, registraram ${ }^{(21)}$ :

- pega medular mais rápida no grupo de CTP;

- recaída, mortalidade e sobrevida sem diferença entre os dois grupos, concluindo que CTP de doador não relacionado e HLA compatível pode ser usada como fonte de células progenitoras.

7. Beelen et al. ${ }^{(22)}$, ampliando trabalho apresentado em $1999^{(15)}$, mostraram que a doença residual molecular e citogenética em pacientes de $\mathrm{LMC} \mathrm{Ph} 1+$, em fase crônica, é reduzida nos pacientes que recebem CTP em comparação com a MO. Em 123 pacientes transplantados - 53 CTP e 70 MO houve significância estatística, tanto para a recaída molecular, como para a recaída citogenética. A expectativa de continuar em remissão após cinco anos também foi maior no grupo de CTP, confirmando que o efeito antileucemia da CTP é maior que o da MO para LMC Ph1+ fase crônica ${ }^{(22)}$.

8. Hagglund et al., comparando 70 transplantes realizados por CTP e 70 por MO, entre 1992 e 1999, observaram $^{(23)}$ :

- pega medular mais rápida, menor número de transfusões de plaquetas e menor número de bacteremias no grupo de CTP;

- GVHD, mortalidade e sobrevida semelhantes nos dois grupos. 


\section{DISCUSSÃO E CONCLUSÕES}

Os trabalhos revisados nas secções anteriores mostram que:

1. mesmo tendo $1 \log$ a mais de células $\mathrm{T}$ na CTP, o índice de DECH aguda não é maior nos transplantes de CTP;

2. existe efeito antitumoral, desencadeado pela CTP, nos transplantes para LMC, o que leva a se ter menos recaída, o que poderia existir, também, para outras doenças;

3. apesar de mais freqüente na CTP, a maioria dos casos de $\mathrm{DECH}$ crônica ocorre de novo e responde bem ao tratamento imunossupressor;

4. a sobrevida, a curto prazo, de pacientes que recebem CTP é maior do que aquela dos pacientes que recebem medula óssea, mas, a longo prazo, ainda não foram observadas diferenças significativas.

Fica claro, assim, que o transplante, usando CTP como fonte de células progenitoras, pode ser usado para tratamento de várias doenças hematológicas. A decisão de substituir a utilização de medula óssea por CTP é, no nosso entendimento, ainda difícil. No ano de 2000, aproximadamente um terço de todos os transplantes alogênicos utilizaram CTP, segundo dados do IBMTR. A maioria desses pacientes estava participando de ensaios clínicos, mas há uma tendência ao aumento deste tipo de transplante, mesmo na rotina clínica.

Nos transplantes de não aparentados, facilidades logísticas e a preferência dos doadores pressionam médicos e doadores a optarem pelo uso de células periféricas. A utilização crescente de manipulação "in vitro" das células, tais como depleção de células $\mathrm{T}$ e seleção positiva, torna necessária a obtenção de grandes números de células CD34+, o que só é factível com a utilização de células mobilizadas por fatores de crescimento e coletadas por aféreses de repetição.

Os transplantes com condicionamento não mieloablativos necessitam de um inóculo de células muito grande e também somente são realizados com células-tronco periféricas. Para transplantes alogênicos entre aparentados, ainda inexistem argumentos sólidos, a favor ou contra a substituição de medula óssea por CTP. Os dois estudos randomizados, prospectivos, grandes e metodologicamente comparáveis, realizados em Seattle, USA ${ }^{(24)}$ e Canadá (Kouban, comunicação pessoal), mostraram vantagens para os grupos que foram transplantados com CTP, nos as- pectos de: menor mortalidade relacionada ao transplante, melhor sobrevida global e livre de doença, principalmente nos pacientes com doenças avançadas e agressivas, mesma incidência de GVHD aguda e tendência ao aumento de GVHD crônico. O estudo europeu (Schmitz, comunicação pessoal), também randomizado, multicêntrico e com um grande número de pacientes, mostrou um aumento da ocorrência de GVHDa e de GVHDc muito mais do que todos os outros estudos publicados até então, mas tal protocolo utilizava G-CSF pós transplante e omitia a dose do dia 11 de metotrexato, no esquema de profilaxia de GVHD.

A tendência observada até agora, mas não comprovada, ainda, de menor ocorrência de recidivas com o uso de CTP, é outro determinante do entusiasmo com tal modalidade de transplante.

Infelizmente, grandes estudos prospectivos e randomizados não estão ativos no momento, pois os estudos de Seatlle e o estudo cooperativo canadense foram interrompidos, prematuramente, devido à observação de vantagens, em análise de interim, em relação à sobrevivência dos grupos que receberam CTP. $\mathrm{O}$ estudo europeu também foi interrompido e a expectativa é de que maiores informações surjam através de estudos de metaanálise que são esperados, utilizando as bases de dados já existentes.

Certamente, os transplantes alogênicos com CTP vieram para ficar. Será necessário desenvolver estratégias que permitam a manipulação do produto de aférese com vistas à utilização de doses de células T ajustadas. A utilização de depleção seletiva de células T, de DLI e de ajustes no regime de condicionamento, além de melhores estratégias de prevenção de GVHD, são instrumentos importantes para a obtenção de melhores resultados nesse tipo de transplante.

A médio e longo prazos, é necessária uma melhor definição das populações de células-tronco e de células efetoras contidas nos produtos a serem infundidos nos pacientes. A manipulação dessas células deve se dar no sentido de que se obtenha uma pega mais rápida e duradoura, melhor proteção contra as infecções e recidivas e menos GVHD.

A individualização do tratamento, considerando aspectos, tais como: estado e agressividade da doença, características do receptor e doador, fatores de risco para GVHD dentre outros, deve ser enfatizada. Estudos clínicos populacionais exigem a homogeneização de grupos de pacientes e procedimentos, enquanto uma boa assistência médica a pacientes individuais pode exigir decisões terapêuticas personalizadas. 
AZEVEDO W \& RIBEIRO MCC. Sources of hematopoietic stem cells for transplantation. Medicina, Ribeirão Preto, 33: 381-389, oct./dec. 2000.

ABSTRACT: Currently, several types of hematopoietic stem cells (HSC), from different tissues and donors, are available for transplantation to human beings. In this review, we discuss the use of allogeneic HSC harvested directly from the bone marrow or mobilized to the peripheral blood with hematopoietic growth factors. We compared these two types of transplants regarding to engraftment, relapse, acute and chronic graft-versus-host disease and survival. Although there is a clear advantage of PBSCT over BMT in the early posttransplant period, higher frequency of cGVHD in PBSCT and relatively short followup of patients do not recommend generalized adoption of allogeneic HSCT so far.

UNITERMS: Bone Marrow Transplantation. Hematopoietic Stem Cell. Hematopoietic Cell Growth Factors.

\section{REFERÊNCIA BIBLIOGRÁFICA}

1 - RIZZO JD. Currents trends in BMT . ABMTR Newsletter 5: 4, 1998

2 - TO LB; ROBERTS MM; HAYLOCK DN; DYSON PG; BRANDFORD AC; THORP D; HO JO; DART GW; HORVATH N \& DAVY NL. Comparision of haematological recovery times and supportive care requirements of autologous peripheral blood stem cell transplants, autologous bone marrow transplants and allogeneic bone marrow transplants. Bone Marrow Transplant 9: 277-284, 1992

3 - CHAMPLIN RE; SCHMITZ N; HOROWITZ MM E; CHAPMS B; CHOPRA R; COMILISSEN JJ; GALE RP; GOLDMAN JM; LOBERIZA FR JR; HERTENSTEIN B; KLEIN JM; MONTSERRAT J; ZHANG MJ; RINGDEN O; TOMANY SC; ROWLINGS PA; VAN HOEF ME \& GRATWOHL A. Blood stem cells compared with bone marrow as a source of hematopoietic cells for allogeneic transplantation. Blood 95:3702-3709, 2000

4 - VIGORITO AC; AZEVEDO WM; MARQUES JFC; AZEVEDO AM; EID KA; ARANHA FJ; LORAND-METZE I; OLIVEIRA GB; CORREA ME; REIS AR; MIRANDA EC \& DE SOUZA CA. A randomised, prospective comparison of allogeneic bone marrow and peripheral blood progenitor cell transplantation in the treatment of haematological malignancies. Bone Marrow Transplant 22:1145-1151, 1998

5 - HELDAL D; TJONNFJORD G; ALBRECHTSEN D; EGELAND T; STEEN R; SOLHEIM BG \& EVENSEN SA. A randomised study of allogeneic transplantation with stem cells from blood or bone marrow. Bone Marrow Transplant 25:1129-1136, 2000

6 - AZEVEDO WM; ARANHA FJ; GOUVEA JV; VIGORITO AC; MARQUES JF Jr; EID KA; AZEVEDO AM \& SOUZA CA. Allogeneic transplantation with blood stem cells mobilized by rhGCSF for haematological malignancies. Bone Marrow Transplant 16:647-653, 1995.

7 - RUSSELL JA; LARRAT L; BROWN C; TURNERAR; CHAUDHRY A; BOOTH K; WOODMAN RC; WOLFF J; VALENTINE K; STEWART D; RUETER JD; RUETHER BA; KLASSEN J; JONES AR; GYONYOR E; ENGLER M; DUNSMORE J;DESAI S; COPPES
MJ; BROWEN T; ANDERSON R \& POON MC. Allogenic blood stem cell and bone marrow transplantation for acute myelogenous leukemia and mielodysplasia: influence of stem cell source on outcome. Bone Marrow Transplant 24:11771183, 1999

8 - LEVINE JE; WIILEY J; KLETZEL M; YANIK G; HUTCHINSON RJ; KOEHLER M \& NEURODRF S. Cytokine-mobilezed allogeneic peripheral blood stem cell transplants in children result in rapid engraftment and a high incidence of chronic GVHD. Bone Marrow Transplant 25:13-18, 2000

9 - RINGDENO; HOROWITZMM; SONDEL P;BIGGS JC; CHAMPLIN RE; DEEG HJ; DICKE K; MASAOKA T \& POWLES RL. Methotrexate, cyclosporine or both to prevent graft-versus-host disease after HLA-identical sibling bone marrow transplantation for early leukemia? Blood 81: 1094-1101, 1993

10 - GYGER M; STUART RK \& PERREAULT C. Immunobiology of allogeneic pripheral blood mononuclear cells mobilized with granulocyte-colony stymulating factor. Bone Marrow Transplant 26:1-16, 2000

11 - ZENG D; DEJBAKHSH-JONES S \& STROBER S. Granulocyte-colony stimulating factor reduces the capacity of blood mononuclear cells to induce graft-versus-host disease: impact on blood progenitor cell transplantation. Blood 19:453463, 1997

12 - APPELBAUM FR. Choosing the source stem cells for allogeneic transplantation: no longer a peripheral issue. Blood 94: 381-383,1999

13 - PRZEPIORKAD; SMITHTL; FOLLODN J; KHOURI I; UENO NT; MEHRA IM; HUG YO; GIRALT S; GAJEWSKI J; DONATO M; CLEARY K; CLAXTON D: BRAUNSCHWEIG I, VAN BESIEN K; ANDERSSON BS, ANDERLINI P \& CHAMPLAIN R. Risk factors for acute graft-versus-host disease after allogeneic blood stem cell transplantation. Blood 94:1465-1470, 1999.

14 - KORBLING M; MIRZA N \& THALL. P100 HLA identical allogeneic blood stem cell transplantation: The MD Anderson Cancer Center experience. Biol Blood Marrow Transplant (abstr 572), 1997. Suppl.1. 
15 - ELMAAGACLI AH; BEELEN DW; OPALKA B SEEBER S \& SCHAEFER UW. The risk of residual molecular and cytogenetic disease in patients with Philadelphia-cromosome positive first chronic myilogenous leukemia is educed after transplantation of allogeneic peripheral blood stem cell compared with bone marrow. Blood 94: 384-389, 1999

16 - SIMPSON DR; COUBAN S; BREDESON $C$ et al. A ramdomized (CBMTG) study comparing peripheral blood ( PB ) and bone marrow ( $B M$ ) in patients undergoing matched sibling transplants for myiloid malignancies. In: Abstract Book. $3^{\text {rd }}$ International Symposium on Allogeneic Blood Progenitor Cell Transplantation, p. 27, 2000

17 - BENSINGER WI; MARTIN P; STORER B et al. A prospective randomized trial of allogeneic peripheral blood stem cells or bone marrow for patients undergoing transplant for the treatment of haematologic malignancies. In: Abstract Book. $3^{\text {rd }}$ International Symposium on Allogeneic Blood Progenitor Cell Transplantation, p. 262000

18 - HELDAL D; TJONNFJORD G; BRINCH L et al. A randomized study of allogeneic transplantation with stem cells from blood or bone marrow. In: Abstract Bok. $3^{\text {rd }}$ International Symposium on Allogeneic Blood Progenitor Cell Transplantation, p.27, 2000

19 - VIGORITO AC; AZEVEDO WM; MARQUES JFC et al. A randomized, prospective comparison of allogeneic bone marrow and peripheral blood progenitor cell transplantation in the treatment of haematological malignancies. In: Abstract Book. $3^{\text {rd }}$ International Symposium on Allogeneic Blood Progenitor Cell Transplantation, p. 27, 2000
20 - GUARDIOLA PH; RUNDE V; BACIGALUPO A et al. Comparison of bone marrow and PBPC for allogeneic SCT in myelodysplastic syndromes. A retrospective study on behalf of the EBMT. In: Abstract Book. $3^{\text {rd }}$ International Symposium on Allogeneic Blood Progenitor Cell Transplantation, p. 29,2000

21 - RINGDEN O; REMBERGER M; BLAU IW et al. Peripheral blood progenitor cells from unrelated donors. In: Abstract Book. $3^{\text {rd }}$ International Symposium on Allogeneic Blood Progenitor Cell Transplantation, p. 38, 2000

22 - BEELEN DW; ELMAAGLACI AH; PENECY R et al. In: Abstract Book. $3^{\text {rd }}$ International Symposium on Allogeneic Blood Progenitor Cell Transplantation, p. 38, 2000

23 - HAGGLUND H, ASCHAN J, BARKHOLT L et al. Lower incidence of bacteremias using allogeneic peripheral blood stem cells ( PBSC ) compared to bone marrow (BM).In: Abstract Book. $3^{\text {rd }}$ International Symposium on Allogeneic Blood Progenitor Cell Transplantation, p. 42, 2000.

24 - BENSINGER WI; MARTIN PJ; STORER B; CLIFT R; FORMAN SJ; NEGRIN R; KASHYAP A; FLOWERS ME; LIBBEBY K; CHAUNCEY TR; STORB R \& APPELBAUM FR. Transplantation of bone marrow as compared with peripheral blood cells from HLA-identical relatives in patients with hematological cancers. N Engl J Med 344: 175-181, 2001.

Recebido para publicação em 25/10/2000

Aprovado para publicação em 20/12/2000 